\title{
ANÁLISIS DE SANIDAD VEGETAL DE CULTIVOS PRÓXIMOS AL DISTRITO DE RIEGO ASUDRA DEL MUNICIPIO DE ÁBREGO - NORTE DE SANTANDER, A PARTIR DE IMÁGENES DE DRONE
}

\section{ANALYSIS OF VEGETABLE HEALTH OF NEXT CROPS TO THE DISTRICT OF IRRIGATION ASUDRA OF THE MUNICIPALITY OF ABREGO - NORTH OF SANTANDER, A PARTY OF IMAGES OF DRONE}

\author{
Ing. Juan David Herrera Galviz*, MSc. Juan Carlos Hernández Criado* \\ * Universidad Francisco de Paula Santander Ocaña, Facultad de Ciencias Agrarias y del \\ Ambiente, Grupo de Investigación GI@DS. \\ Vía Acolsure, Sede El Algodonal, Ocaña, Norte de Santander, Colombia. \\ Teléfono: (57) (7) 5690088 ext. 448. \\ E-mail: $\{$ jdherrerag, jchernandezc $\} @$ ufpso.edu.co.
}

\begin{abstract}
Resumen: El objetivo de este trabajo fue realizar un diagnóstico de la sanidad vegetal de las áreas de cultivo más próximas al distrito de riego ASUDRA, a partir del cálculo del índice de vegetación de diferencia normalizada NDVI. En este sentido, se emplearon recursos tales como un equipo drone para la captura de imágenes aéreas y software para el procesamiento de las imágenes capturadas, así como software SIG, para el cálculo del índice anteriormente citado. Mediante los mapas generados, se evidenció que aunque los terrenos poseen buena disponibilidad de agua, existen otros factores que están afectando el buen desarrollo de la vegetación principalmente en el sector 1 del distrito ubicado al suroriente del casco urbano del municipio de Ábrego, Norte de Santander. En general, la sanidad vegetal favorable alcanza a cubrir aproximadamente el $30 \%$ de las áreas cultivables, cuya superficie total de 79.02 hectáreas, lo que determina que el restante $70 \%$ debe ser analizado a detalle con el fin de encontrar las razones por las que la vegetación de los cultivos presentes en estas áreas, no poseen una sanidad vegetal favorable. Y en este sentido, se deben enfocar esfuerzos para diagnosticar los suelos de esta zona y formular estrategias más precisas de agricultura de precisión, con el fin de hacer un uso y ahorro eficiente del agua, y aumentar la productividad.
\end{abstract}

Palabras clave: NDVI, distrito de riego ASUDRA, drone, procesamiento de imágenes, sanidad vegetal.

Abstract: The objective of this work was to make a diagnosis of the plant health of the crop areas closest to the ASUDRA irrigation district, based on the calculation of the NDVI standardized difference vegetation index. In this sense, resources such as drone equipment were used for the capture of aerial images and software for the processing of the captured images, as well as GIS software, for the calculation of the aforementioned index. Through the generated maps, it was evidenced that although the lands have good water availability, there are other factors that are affecting the good development of vegetation mainly in sector 1 of the district located to the southeast of the town center of Ábrego, Norte de Santander. In general, favorable plant health reaches approximately $30 \%$ of the arable areas, whose total area is 79.02 hectares, which determines that the remaining $70 \%$ must be analyzed in detail in order to find the reasons why vegetation of the crops present in these areas, do not have a favorable plant health. And in this sense, efforts should be focused to diagnose the soils of this area and formulate more precise 
precision agriculture strategies, in order to make efficient use and saving of water, and increase productivity.

Keywords: NDVI, ASUDRA irrigation district, drone, image processing, plant health.

\section{INTRODUCCIÓN}

La agricultura de precisión es considerada un grupo de técnicas para planificar el uso de agroquímicos y del agua teniendo en cuenta la diversidad del medio físico y biológico, con el fin de optimizar costos de producción y mejorar la eficiencia de los cultivos, disminuyendo los impactos ambientales adversos (Burgos-Artizzu, Ribeiro, \& De Santos, 2007). Así, existen autores que han abordado en sus trabajos, el uso de la tecnología de percepción remota para capturar información relacionada con los factores que afectan la producción de las plantas en un cultivo como lo son el índice de área foliar (LAI), verdor de la hoja, altura, nitrógeno en la hoja, contenido de clorofila, o contenido de agua entre otros, que se pueden determinar mediante distintas técnicas, dentro de las que resaltan los índices de vegetación (Kharuf-Gutierrez, Hernández-Santana, OrozcoMorales, de la C. Aday-Díaz, \& Delgado-Mora, 2018); (C Ferrin et al., 2017).

Dentro de los índices de vegetación más utilizados en agricultura de precisión, se encuentra el índice de Vegetación de Diferencia Normalizada o NDVI. Este índice se basa en la reflexión diferencial de la vegetación en las bandas roja e infrarroja cercana, y se puede usar como forma de monitoreo remoto de la vegetación, tanto en tiempo como en espacio (Salinas-Zavala, Martínez-Rincón, \& MoralesZárate, 2017) (Gonzaga-Aguilar, 2015), aunque hay que tener en cuenta que este índice no utiliza un parámetro de ajuste que varía según la densidad de la vegetación (Tello, Gómez-Báguena, \& Casterad, 2017), por lo que la información capturada y procesada, se debe complementar con el cálculo de otros índices de vegetación. Hoy día, la tecnología drone ha tomado relevancia en la temática de agricultura de precisión, evidenciándose sus aplicaciones principalmente en la captura de información para realizar análisis relacionados con los índices de vegetación (Gillan, McClaran, Swetnam, \& Heilman, 2019)(Maes \& Steppe, 2019)(Fischer, Greiner, Lutman, Webber, \& Vercauteren, 2019), y así poder planificar el uso de los insumos agroquímicos y el riego, en donde también se conoce que los drones pueden actuar, principalmente como herramientas para esparcir los agroquímicos de forma mesurada, puntual y eficientemente (Mogili \& Deepak, 2018).
En Colombia, es común escuchar la gestión e implementación de infraestructura de distritos de riego, ya que estos se usan para canalizar agua de ríos y arroyos, que luego se distribuye por un área determinada, con el fin de irrigar cultivos, funcionando así hace varias décadas, como infraestructura de carácter público, que a comienzos de los años 90 fueron cedidos a Asociaciones de Usuarios y a Organizaciones privadas (Petzl, Quiroga, Rodríguez, Rojas, \& Ojeda, 2015); (C Hernández et al., 2016).

En la provincia de Ocaña, se encuentra ubicado el municipio de Ábrego en donde funciona el Distrito de Riego ASUDRA, cuya representatividad está justificada teniendo en cuenta su antigüedad, la cantidad de usuarios que beneficia, así como el área de influencia del mismo. Este distrito capta agua directamente de los tributarios principales que ayudan a formar el río algodonal. Sin embargo, con el pasar del tiempo, el distrito ha perdido vida útil y algunos tramos de su infraestructura ya están obsoletos, lo que dificulta que toda la infraestructura sea operativa y por consiguiente, dificulta que algunos de sus usuarios puedan acceder al recurso hídrico que se distribuye por el distrito.

Lo anterior genera una problemática de uso ineficiente del recurso hídrico y se traduce en un gran obstáculo para la producción agrícola de los usuarios de ASUDRA, lo que dificulta incorporar técnicas de agricultura de precisión (AP) para optimizar la planificación de sus cultivos. De acuerdo a lo anterior, este trabajo realiza el cálculo y espacialización del índice de vegetación de diferencia normalizada NDVI, a partir de imágenes capturadas con un vehículo aéreo no tripulado RPAS o drone, en los cuatro sectores del distrito de riego que actualmente se poseen, con el fin de que estos insumos permitan la toma de decisiones con respecto a la planificación de acciones que permitan mejorar la productividad de los suelos, que a su vez se refleje en la sanidad vegetal de las especies vegetales que en estos suelos se cultivan.

\section{MATERIALES Y MÉTODOS}

El desarrollo de este trabajo, se efectuó en el Distrito de Riego ASUDRA ubicado en la zona rural aledaña al casco urbano del municipio de Ábrego - Norte de Santander. Este distrito, se 
divide en 4 sectores, de los cuáles los sectores 2, 3 y 4 se encuentran conectados y distribuyen el agua captada del río frío, mientras el sector 1 se encarga de distribuir el agua captada del río oroque, descargando el agua sobrante, al río frío justo en el mismo punto donde comienza el sector 2 del distrito. Este sector 2, a su vez finaliza en la quebrada el tabaco, al igual que el sector 4 que comienza en la represa de río frío y termina en esta misma quebrada, mientras el sector 3 une los sectores 4 y 2 . En la siguiente figura se puede evidenciar la distribución espacial de la zona de estudio:

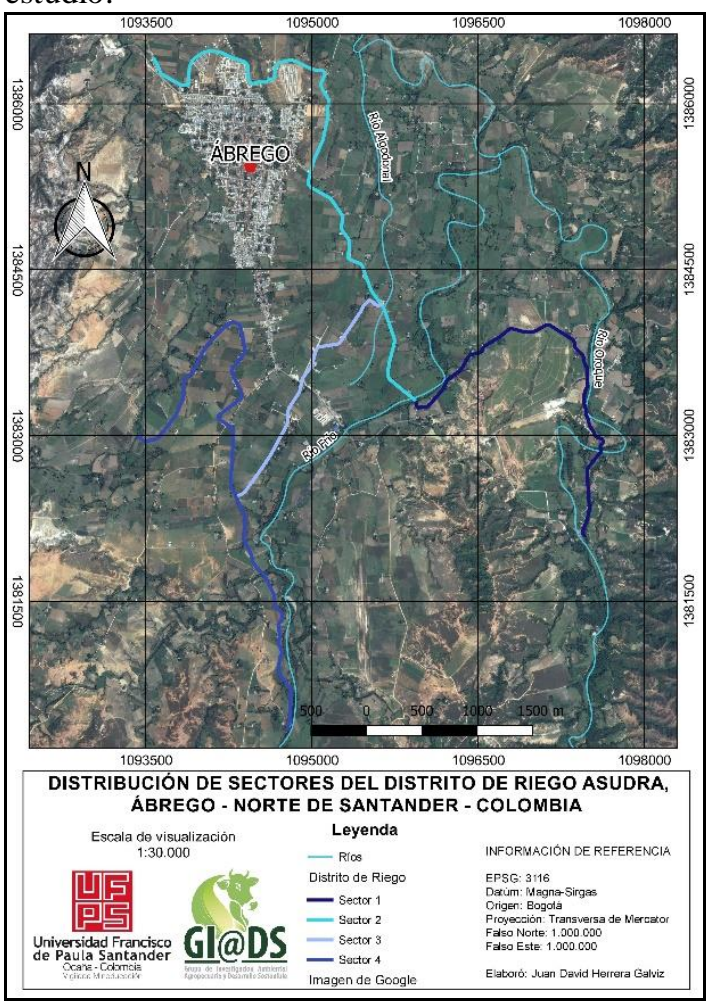

Fig. 1. Zona de estudio. Fuente: Elaboración propia.

Inicialmente se realizó un recorrido de reconocimiento de la zona, identificando que existe una presencia considerable de ganadería en la gran mayoría de predios que se benefician de la infraestructura del distrito (ver figura 2). Así mismo, también se observó que en los predios donde se poseen cultivos agrícolas, las especies seleccionadas corresponden en su mayoría a maíz, plátano y pimentón, y en menor escala: caña de azúcar, fríjol y café. De acuerdo a lo anterior, se procedió a definir la cantidad de vuelos a ejecutar, toda vez que inicialmente se pretendía levantar todo el área aledaño al distrito en vuelos de la misma altura, pero como las condiciones del terreno son diferentes, se pueden emplear vuelos de diferentes alturas dependiendo, el contexto de cada sitio en particular. Los vuelos planificados fueron los siguientes:

Tabla 1. Vuelos planificados y ejecutados

\begin{tabular}{|c|c|c|c|c|}
\hline Lugar & & $\begin{array}{l}\text { No. De } \\
\text { Vuelo }\end{array}$ & $\begin{array}{l}\text { Mes de } \\
\text { realización }\end{array}$ & $\begin{array}{l}\text { Altura en } \\
\text { la que se } \\
\text { realizó }\end{array}$ \\
\hline \multirow{4}{*}{$\begin{array}{l}\text { Sector } \\
\text { del } \\
\text { Distrito }\end{array}$} & 1 & 1 & $\begin{array}{ll}\text { Abril } & \text { de } \\
2018 & \end{array}$ & $\begin{array}{l}122 \\
\text { metros }\end{array}$ \\
\hline & & 2 & $\begin{array}{l}\text { Abril } \\
2018\end{array}$ & $\begin{array}{l}105.8 \\
\text { metros }\end{array}$ \\
\hline & & 3 & $\begin{array}{l}\text { Mayo } \\
2018\end{array}$ & $\begin{array}{l}238.2 \\
\text { metros }\end{array}$ \\
\hline & & 4 & $\begin{array}{l}\text { Mayo } \\
2018\end{array}$ & $\begin{array}{l}238.5 \\
\text { metros }\end{array}$ \\
\hline \multirow{4}{*}{$\begin{array}{l}\text { Sector } \\
\text { del } \\
\text { Distrito }\end{array}$} & 2 & 5 & $\begin{array}{l}\text { Mayo } \\
2018\end{array}$ & $\begin{array}{l}92.2 \\
\text { metros }\end{array}$ \\
\hline & & 6 & $\begin{array}{l}\text { Junio } \\
2018\end{array}$ & $\begin{array}{l}92 \\
\text { metros }\end{array}$ \\
\hline & & 7 & $\begin{array}{l}\text { Junio } \\
2018\end{array}$ & $\begin{array}{l}92.4 \\
\text { metros }\end{array}$ \\
\hline & & 8 & $\begin{array}{l}\text { Junio } \quad \text { de } \\
2018\end{array}$ & $\begin{array}{l}92.6 \\
\text { metros }\end{array}$ \\
\hline \multirow{3}{*}{$\begin{array}{l}\text { Sector } \\
\text { del } \\
\text { Distrito }\end{array}$} & 3 & 9 & Julio de 2018 & $\begin{array}{l}93 \\
\text { metros }\end{array}$ \\
\hline & & 10 & Julio de 2018 & $\begin{array}{l}243 \\
\text { metros }\end{array}$ \\
\hline & & 11 & Julio de 2018 & $\begin{array}{l}92.4 \\
\text { metros }\end{array}$ \\
\hline \multirow{3}{*}{$\begin{array}{l}\text { Sector } \\
\text { del } \\
\text { Distrito }\end{array}$} & 4 & 12 & $\begin{array}{l}\text { Agosto } \\
2018\end{array}$ & $\begin{array}{l}123.8 \\
\text { metros }\end{array}$ \\
\hline & & 13 & $\begin{array}{l}\text { Agosto } \\
2018\end{array}$ & $\begin{array}{l}205.4 \\
\text { metros }\end{array}$ \\
\hline & & 14 & $\begin{array}{l}\text { Agosto } \\
2018\end{array}$ & $\begin{array}{l}204 \\
\text { metros }\end{array}$ \\
\hline
\end{tabular}

Fuente: elaboración propia.

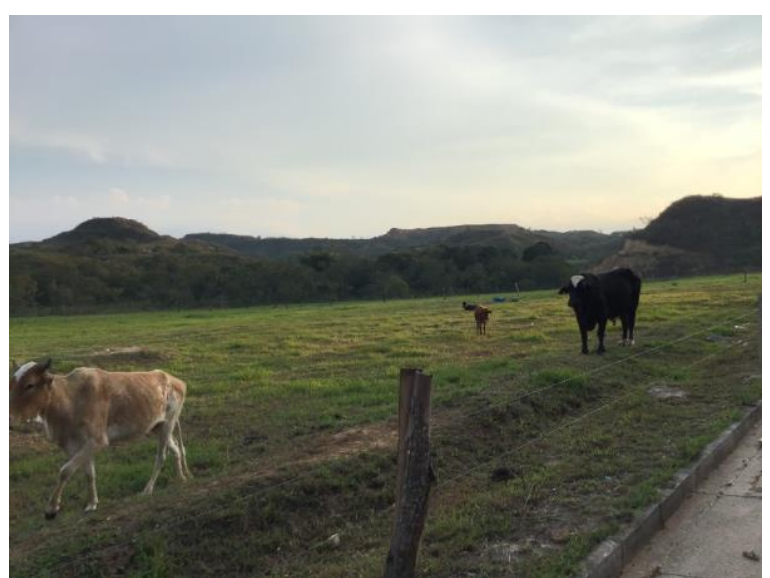

Fig. 2. Ganadería en la zona de estudio. Fuente: Elaboración propia 
Los vuelos planificados en la Tabla 1, fueron ejecutados tal cual se planificaron, y para ello se empleó un equipo drone DJI Phantom 3 Advanced de la Universidad Francisco de Paula Santander Ocaña (Ver figura 3)

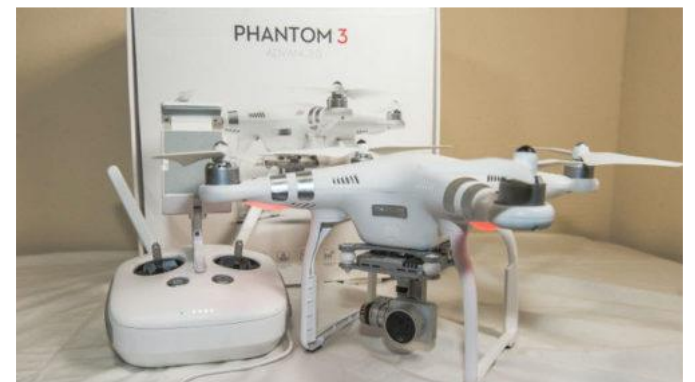

Fig. 3. Drone empleado para el trabajo. Fuente: RedShark.

Es importante resaltar que para la captura de imágenes aéreas con drone, para fines cartográficas, es necesario establecer primero la planimetría de cada vuelo, empleando alguna aplicación eficiente para tal finalidad. Así, se empleó la aplicación del fabricante del equipo, llamada DJI GS PRO (Ver figura 4), que funciona por el momento, sólo en dispositivos Apple.

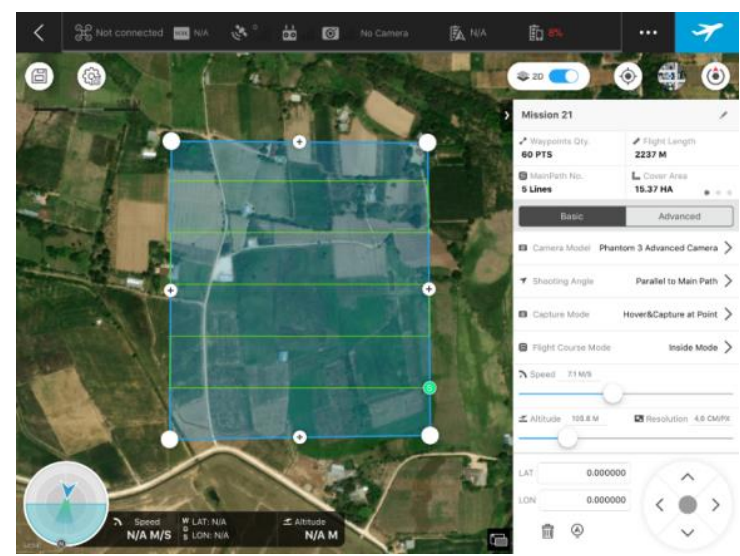

Fig. 2. Ejemplo de planimetría de un vuelo en DJI GS Pro. Fuente: Elaboración propia.

Después de ejecutados cada uno de los 14 vuelos planificados, se realizó el procesamiento de las imágenes capturadas por cada vuelo, usando el software Pix4D, del cual la Universidad posee una licencia indefinida. De cada vuelo, el software generó el respectivo mosaico y el modelo digital de elevación. Posteriormente, en el software QGIS, se realizó el cálculo del índice Índice de vegetación de diferencia normalizada (NDVI), usando la siguiente ecuación (Gonzaga-Aguilar, 2015):
NDVI:

$\mathrm{NIR}=$ reflectancia corregida atmosféricamente correspondiente al infrarrojo cercano, que corresponde a la Banda 8A en Sentinel.

$\mathrm{R}=$ reflectancia corregida atmosféricamente correspondiente al rojo, que corresponde a la banda 4 en Sentinel.

$\mathrm{NDVI}=\frac{N I R-R}{N I R+R}$

Así se obtuvieron 14 mapas de NDVI, a partir de los cuáles se realizó el respectivo análisis de lo evidenciado, con el fin de realizar unas recomendaciones claras y concisas a los agricultores usuarios del distrito de riego ASUDRA.

\section{RESULTADOS Y DISCUSIÓN}

Los 14 vuelos ejecutados, permitieron capturar 855 imágenes en total, para un área aproximada de 340 hectáreas, a partir de las cuáles se delimitaron 177 cultivos que corresponden a un área de 79.02 hectáreas. A continuación se visualizan cada uno de los mapas generados, en las figuras 5-18:

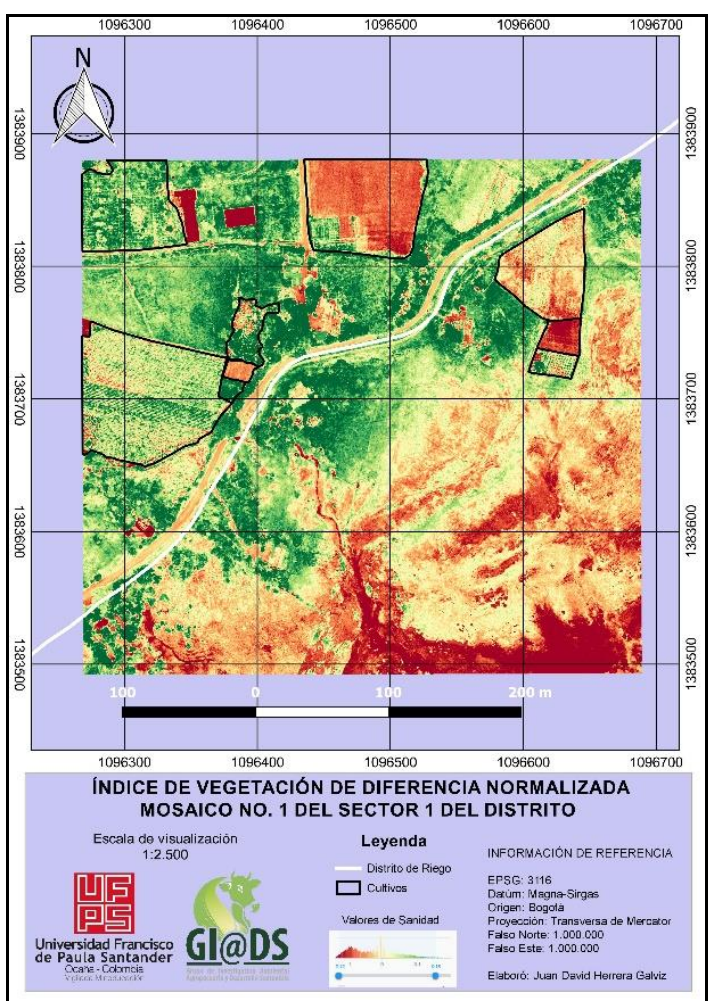

Fig. 5. NDVI Mosaico 1 del Sector 1 del Distrito. Fuente: Elaboración propia. 


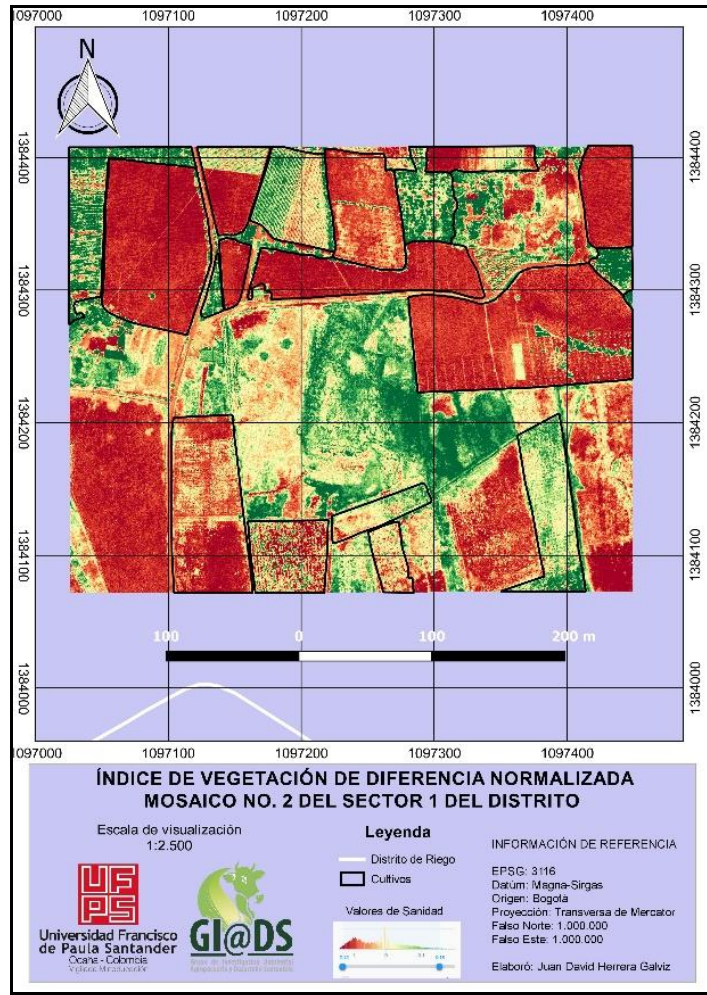

Fig. 6. NDVI Mosaico 2 del Sector 1 del Distrito. Fuente: Elaboración propia.

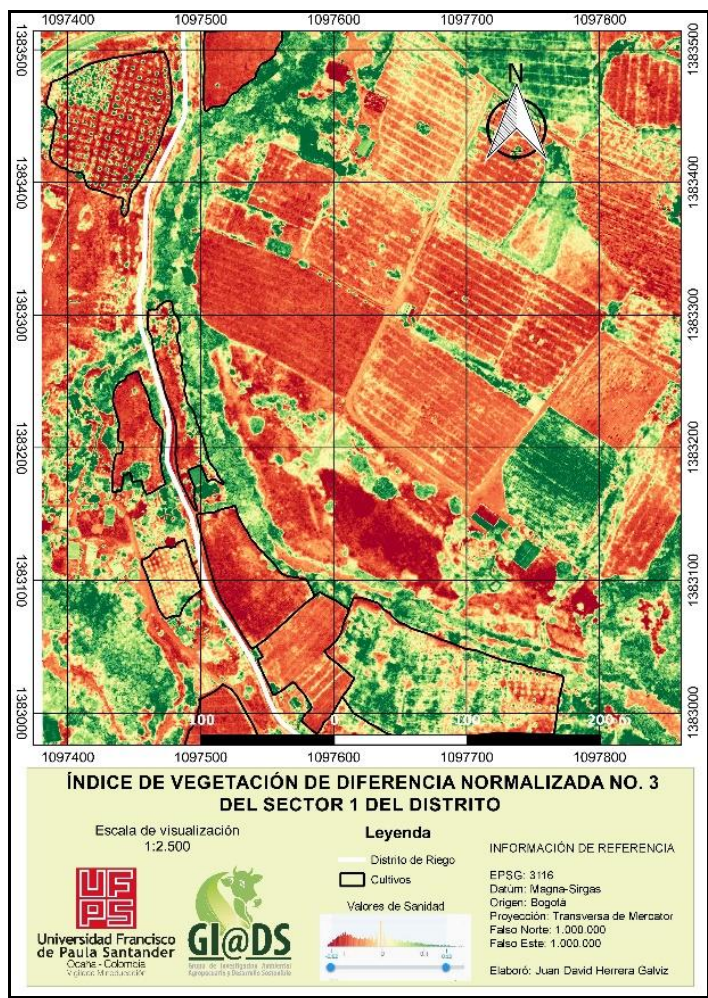

Fig. 7. NDVI Mosaico 3 del Sector 1 del Distrito. Fuente: Elaboración propia.

Universidad de Pamplona I. I. D. T. A.

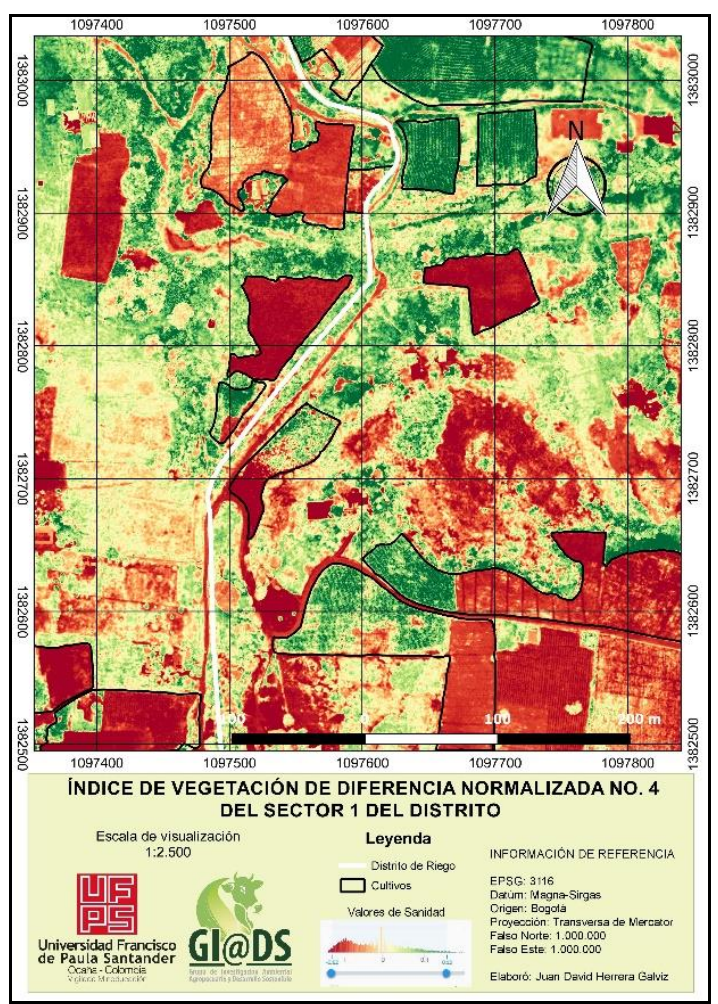

Fig. 8. NDVI Mosaico 4 del Sector 1 del Distrito. Fuente: Elaboración propia.

En las figuras 5 - 8, se evidencian los NDVI de los vuelos 1, 2, 3 y 4 del sector 1 del distrito de riego. En el caso del mosaico 1, se visualiza que de los nueve cultivos delimitados, sólo dos, ubicados al noroeste del área de estudio, presentan una vegetación sana por lo menos en un $80 \%$ de su superficie. En este sentido es importante aclarar que el único cultivo ubicado en la parte norte céntrica del mapa, presenta baja sanidad vegetal, debido a que en dichas áreas se estaban realizando en su momento, trabajos de labranza para preparación del terreno a cultivar.

En el mosaico 2, se visualiza que de los 18 cultivos delimitados, sólo tres, dos de ellos ubicados en la zona norte y uno ubicado en la zona sureste, presentan una vegetación sana por lo menos en un $50 \%$ de su superficie. En este sentido es importante aclarar que aproximadamente cinco cultivos ubicados en la parte norte del mapa, presenta baja sanidad vegetal, debido a que en dichas áreas se estaban realizando en su momento, trabajos de labranza para preparación del terreno a cultivar.

En el mosaico 3, se visualiza que de los ocho cultivos delimitados, sólo uno, presenta una vegetación sana por lo menos en un $50 \%$ de su 
superficie. En este sentido es importante aclarar que aproximadamente tres cultivos ubicados al oeste del distrito, presenta baja sanidad vegetal, debido a que en dichas áreas se estaban realizando en su momento, trabajos de labranza para preparación del terreno a cultivar. (L Tangarife et al., 2017).

En el mosaico 4, se visualiza que de los 12 cultivos delimitados, sólo dos, presenta una vegetación sana por lo menos en un $85 \%$ de su superficie. En este sentido es importante aclarar que aproximadamente siete cultivos ubicados en este mapa, presenta baja sanidad vegetal, debido a que en dichas áreas se estaban realizando en su momento, trabajos de labranza para preparación del terreno a cultivar.

En los otros cultivos de los 4 mosaicos, es necesario desarrollar análisis de suelos para detectar la causa que genera tan baja sanidad en la vegetación con menos valores de NDVI reflejados en los mapas.

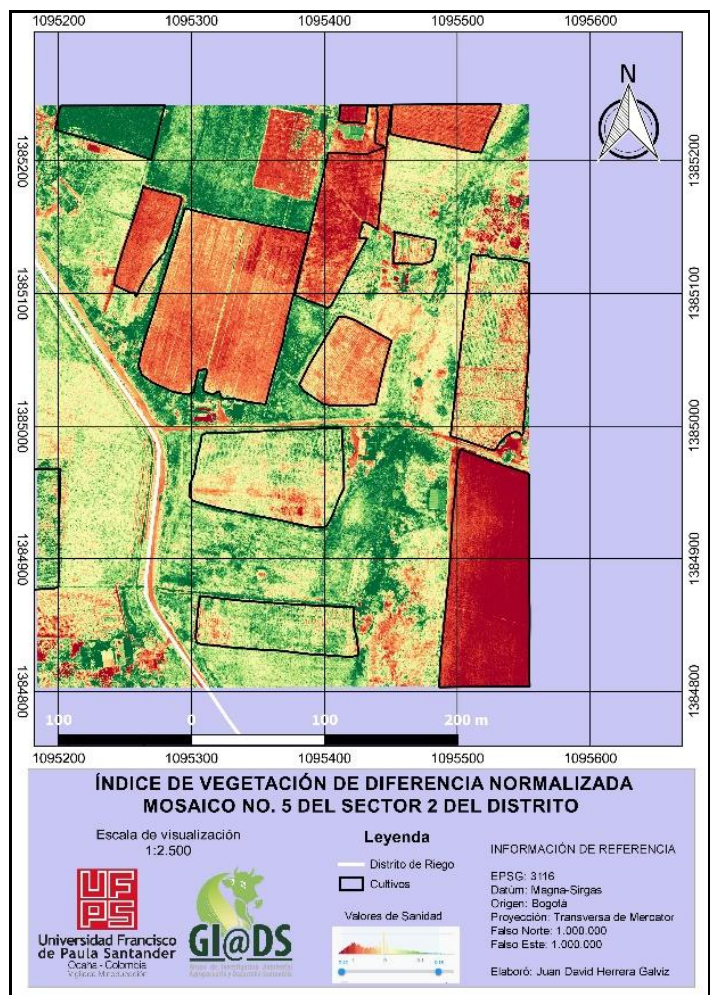

Fig. 9. NDVI Mosaico 5 del Sector 2 del Distrito. Fuente: Elaboración propia.

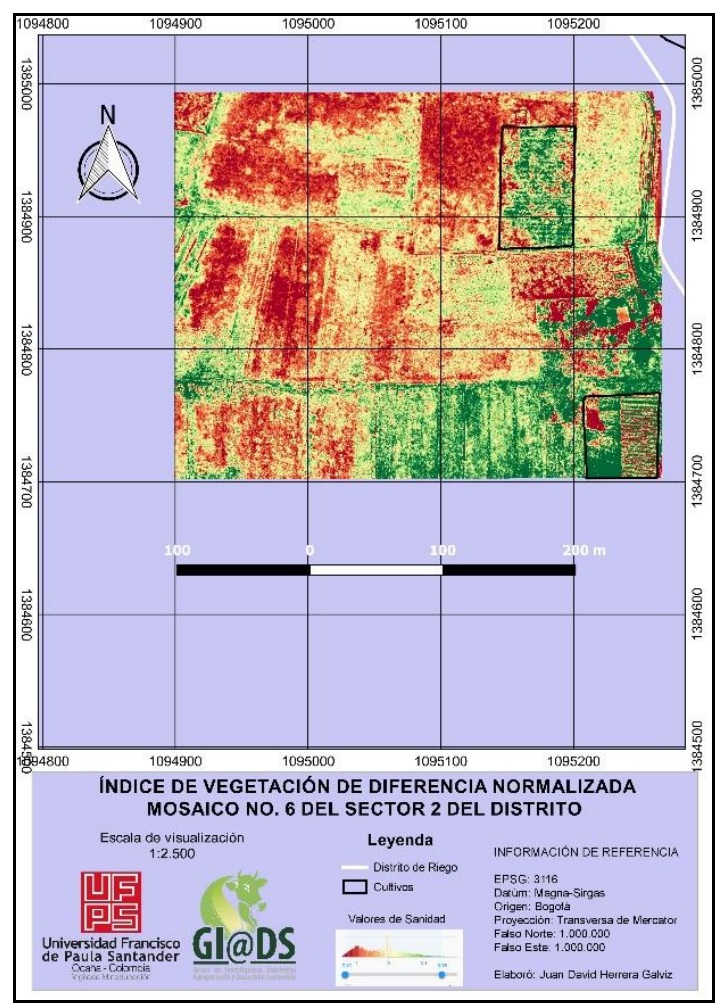

Fig. 10. NDVI Mosaico 6 del Sector 2 del Distrito. Fuente: Elaboración propia.

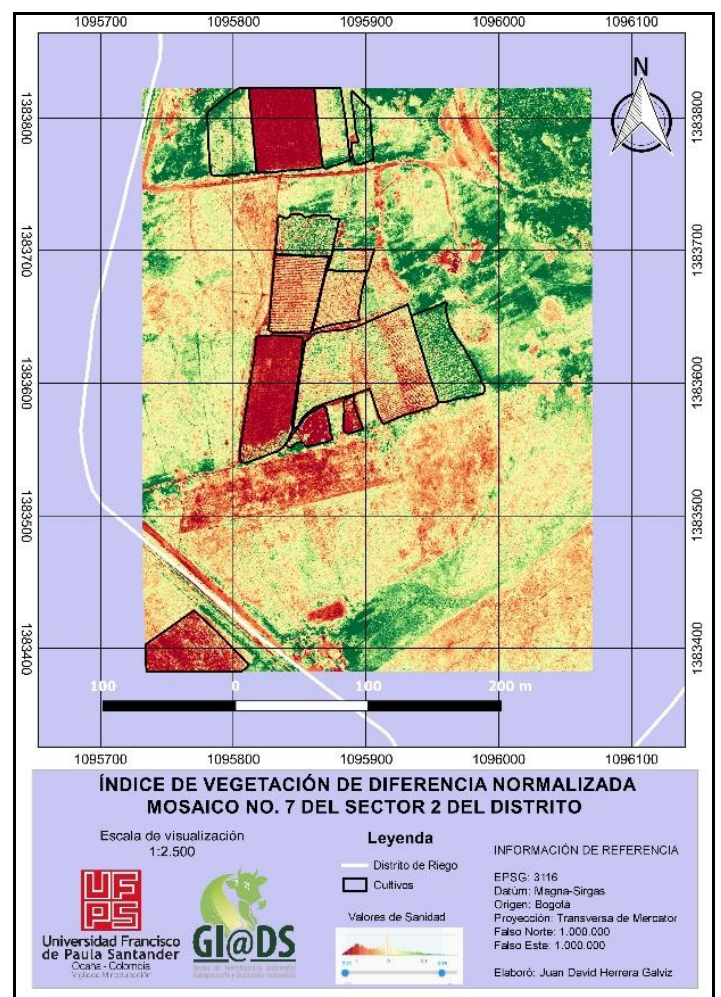

Fig. 11. NDVI Mosaico 7 del Sector 2 del Distrito. Fuente: Elaboración propia. 


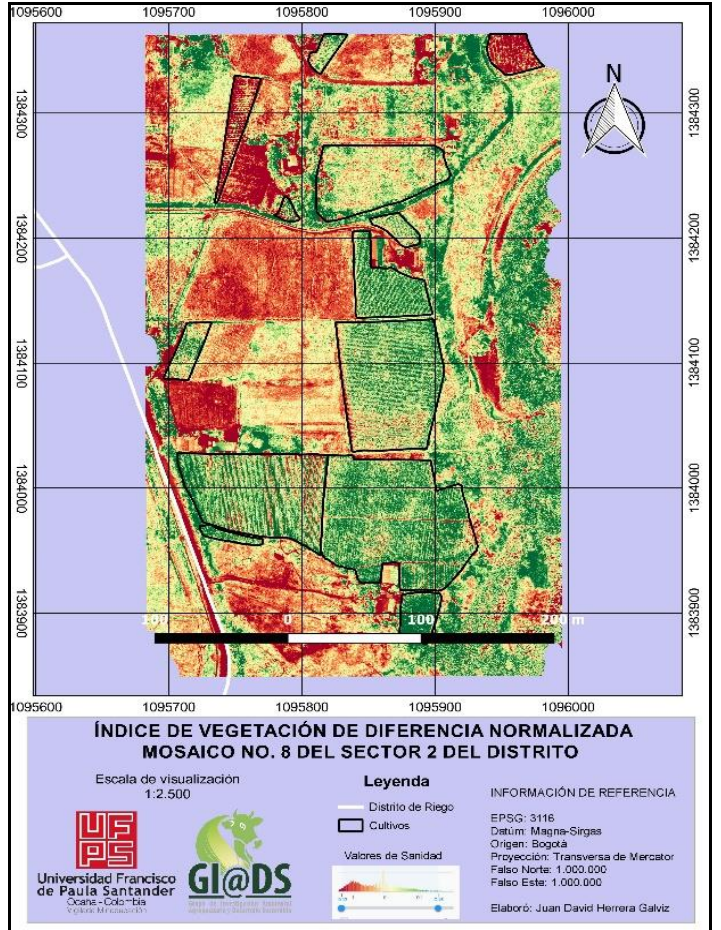

Fig. 12. NDVI Mosaico 8 del Sector 2 del Distrito. Fuente: Elaboración propia.

En la figura 9-12, se visualizan los vuelos 5, 6, 7 y 8 correspondientes al sector 2 del distrito de riego. En el mosaico 5, se evidencia que de los 12 cultivos delimitados, sólo uno, presenta una vegetación sana por lo menos en un $85 \%$ de su superficie En este sentido es importante aclarar que aproximadamente seis cultivos ubicados en este mapa, presentan baja sanidad vegetal, debido a que en dichas áreas se estaban realizando en su momento, trabajos de labranza para preparación del terreno a cultivar.

En el mosaico 6, se visualiza que en los dos cultivos delimitados, se presenta una vegetación sana por lo menos en un $40 \%$ de su superficie; por lo que se hace necesario desarrollar análisis de suelos para detectar la causa que genera baja sanidad en la vegetación en el $60 \%$ de área restante.

En el mosaico 7, se visualiza que de los 14 cultivos delimitados, sólo dos, presenta una vegetación sana por lo menos en un 50\% de su superficie. En este sentido es importante aclarar que aproximadamente cuatro cultivos ubicados en este mapa, presenta baja sanidad vegetal, debido a que en dichas áreas se estaban realizando en su momento, trabajos de labranza para preparación del terreno a cultivar.

En el mosaico 8, se visualiza que de los 14 cultivos delimitados, sólo seis, presentan una vegetación sana por lo menos en un 55\% de su superficie. En

\section{Universidad de Pamplona}

I. I. D. T. A. los otros cultivos de los 4 mosaicos del sector 2, es necesario desarrollar análisis de suelos para detectar la causa que genera tan baja sanidad en la vegetación con menos valores de NDVI reflejados en los mapas.

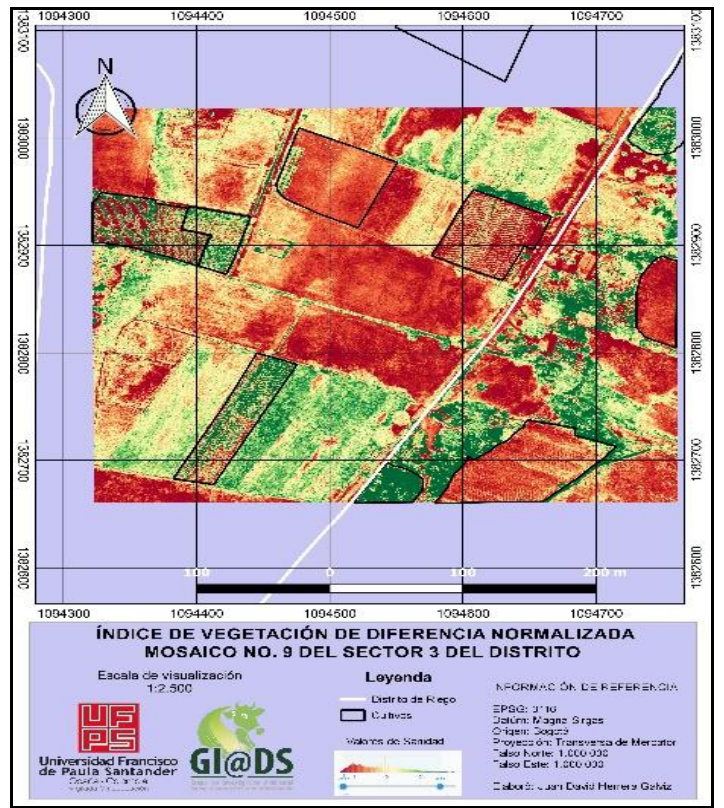

Fig. 13. NDVI Mosaico 9 del Sector 3 del Distrito. Fuente: Elaboración propia.

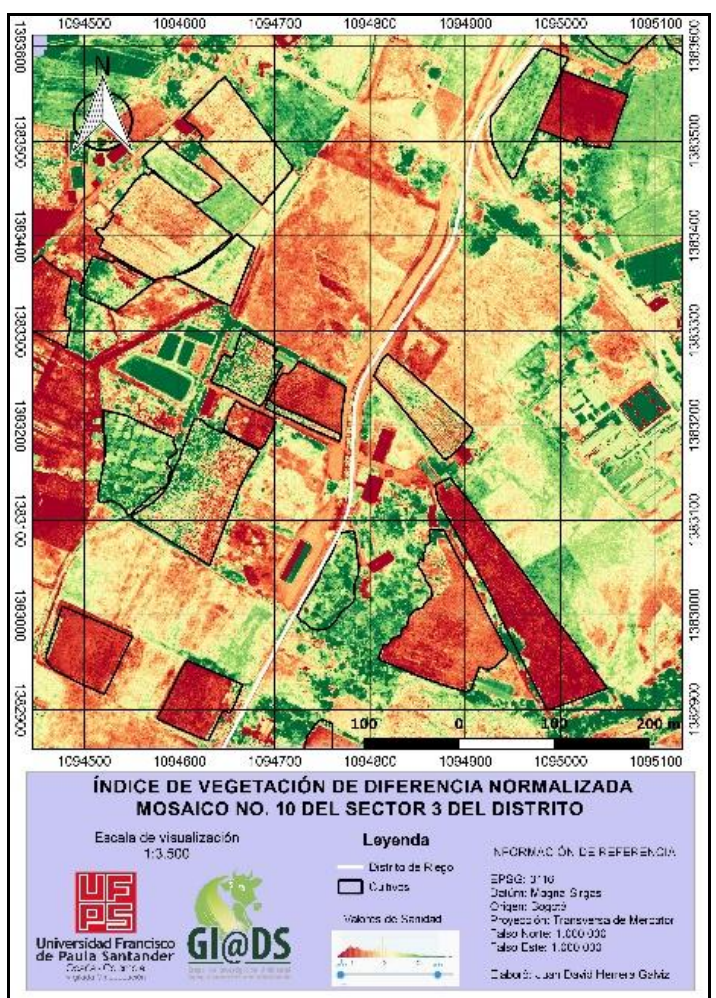


Fig. 14. NDVI Mosaico 10 del Sector 3 del Distrito. Fuente: Elaboración propia.

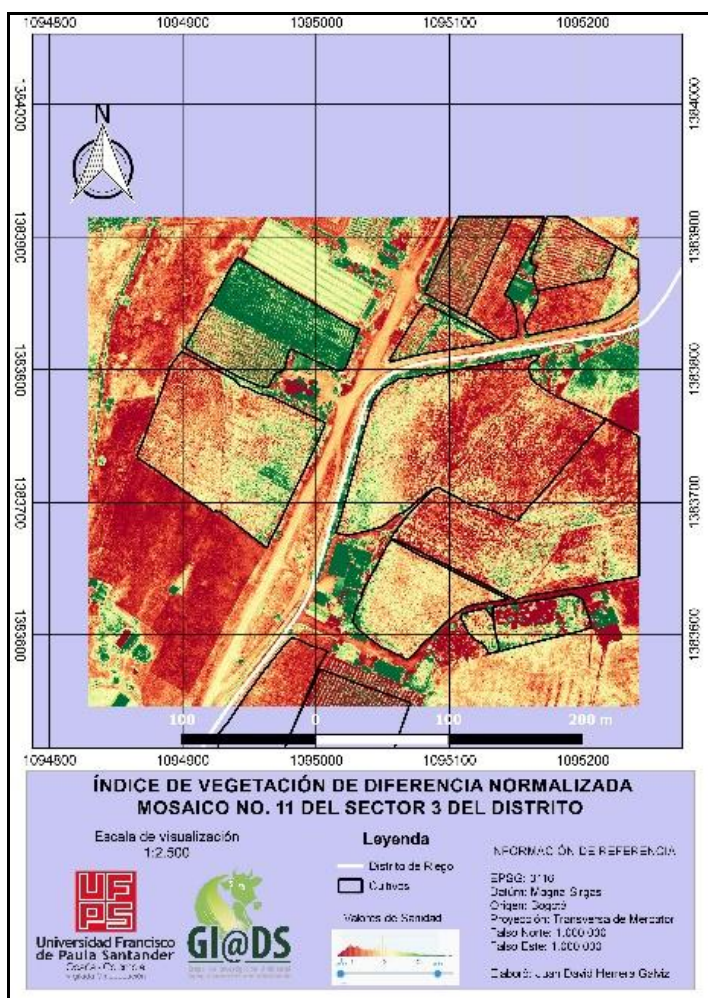

Fig. 15. NDVI Mosaico 11 del Sector 3 del

Distrito. Fuente: Elaboración propia.

En la figura 13-15, se visualizan los vuelos $9,10 \mathrm{y}$ 11, correspondientes al sector 3 del distrito de riego. Así, en el mosaico 9, se visualiza que de los ocho cultivos delimitados, dos presentan una vegetación sana por lo menos un $40 \%$ de su área, mientras uno, presenta dicha característica en más del $80 \%$ de su superficie. En este sentido es importante aclarar que aproximadamente tres cultivos ubicados en este mapa, presentan baja sanidad vegetal, debido a que en dichas áreas se estaban realizando en su momento, trabajos de labranza para preparación del terreno a cultivar.

En el mosaico 10, se visualiza que de los 18 cultivos delimitados, cinco presentan una vegetación sana por lo menos en un $40 \%$ de su área. En este sentido es importante aclarar, que aproximadamente cuatro cultivos ubicados en este mapa, presentan baja sanidad vegetal, debido a que en dichas áreas se estaban realizando en su momento, trabajos de labranza para preparación del terreno a cultivar.

$\mathrm{Y}$ en el mosaico 11, se visualiza que de los 12 cultivos delimitados, sólo un cultivo presenta vegetación sana en el 55\% de su área. En los otros Universidad de Pamplona

I. I. D. T. A. cultivos de los 3 mosaicos del sector 3, es necesario desarrollar análisis de suelos para detectar la causa que genera tan baja sanidad en la vegetación con menos valores de NDVI reflejados en los mapas.

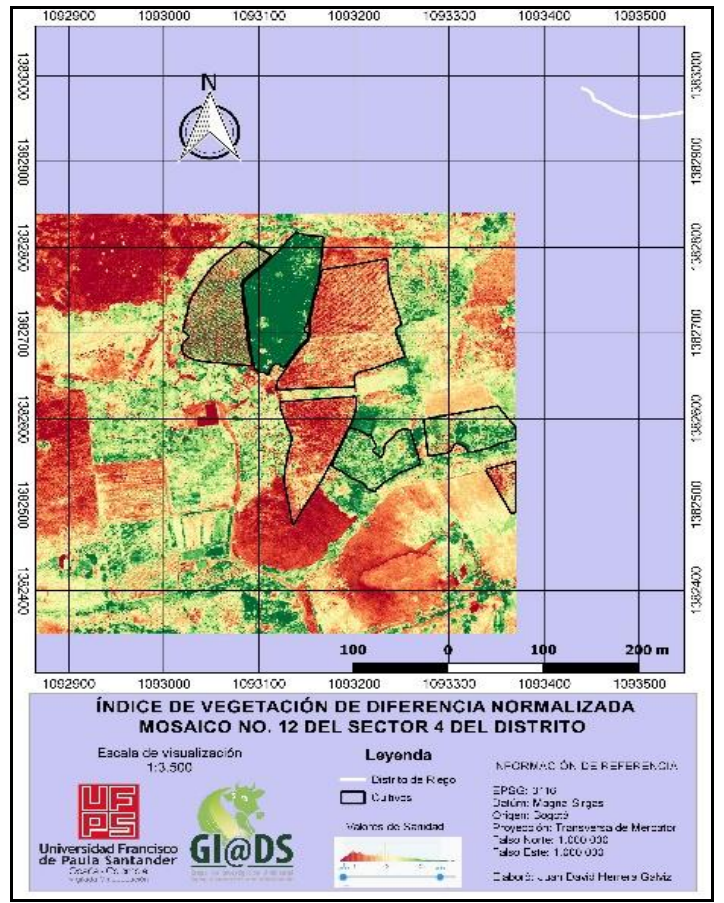

Fig. 16. NDVI Mosaico 12 del Sector 4 del Distrito. Fuente: Elaboración propia.

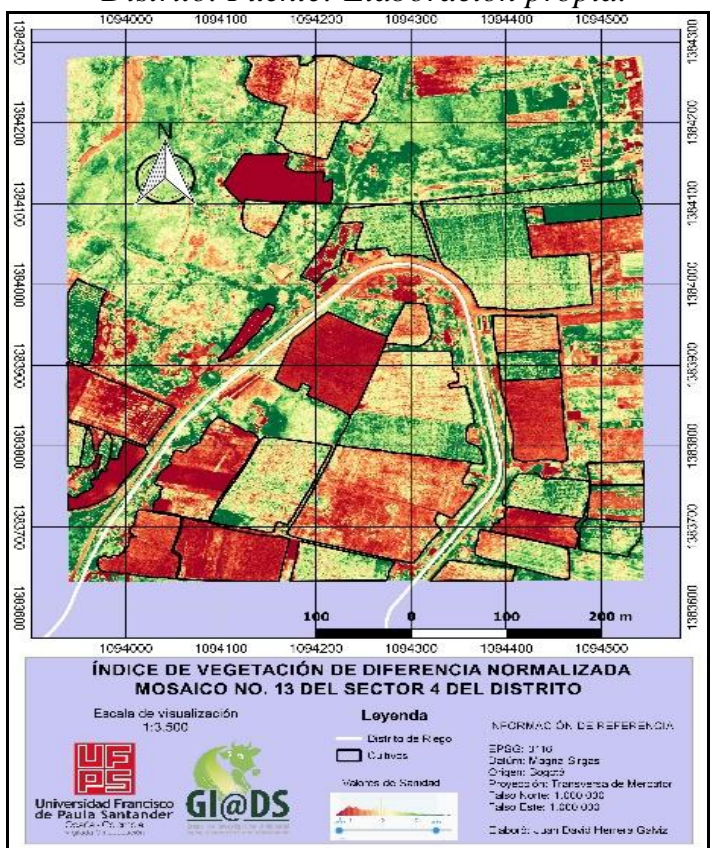

Fig. 17. NDVI Mosaico 13 del Sector 4 del Distrito. Fuente: Elaboración propia. 


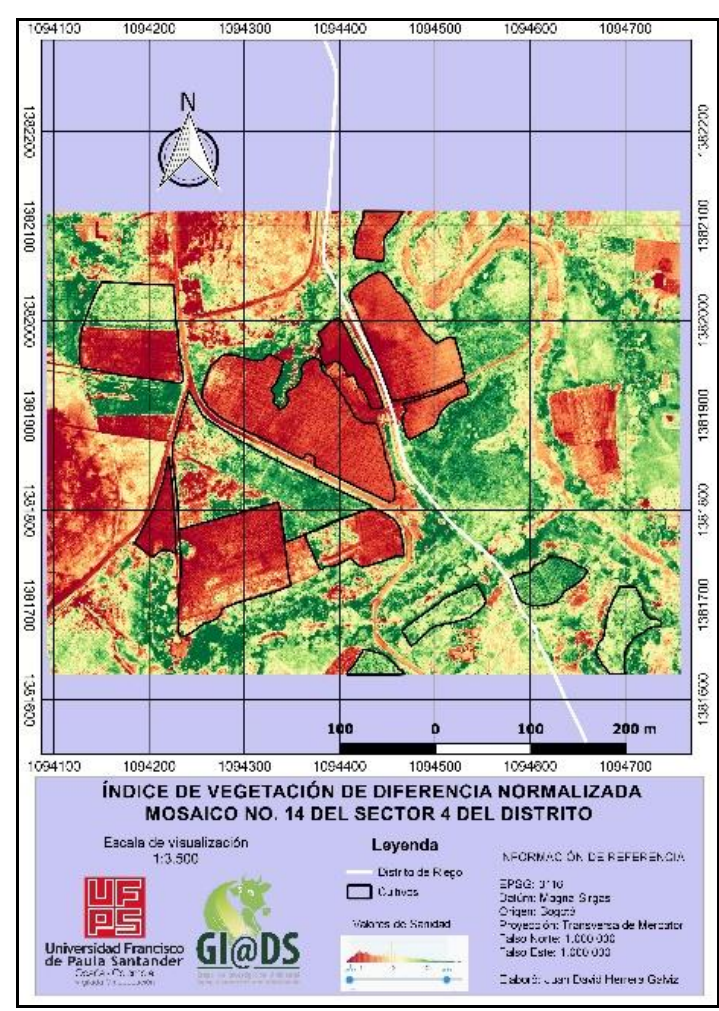

Fig. 18. NDVI Mosaico 14 del Sector 4 del

Distrito. Fuente: Elaboración propia.

En la figura 16-18, se visualizan los vuelos 12,13 y 14 , correspondientes al sector 4 del distrito de riego. En el mosaico 12, se visualiza que de los siete cultivos delimitados, dos presentan una vegetación sana por lo menos en un $40 \%$ de su área, y un cultivo presenta esta misma característica en un $90 \%$ de su superficie, pues corresponde a un cultivo de café entre árboles.

En el mosaico 13, se visualiza que de los 31 cultivos delimitados, ocho presentan una vegetación sana en un $40 \%$ o más de su área. En este sentido es importante aclarar, que aproximadamente siete cultivos ubicados en este mapa, presentan baja sanidad vegetal, debido a que en dichas áreas se estaban realizando en su momento, trabajos de labranza para preparación del terreno a cultivar. (J Plaza, M Núñez, 2017).

$\mathrm{Y}$ en el mosaico 14, se visualiza que de los 14 cultivos delimitados, cuatro cultivos ubicados al Sur del mapa presentan una vegetación sana, por lo menos en un $40 \%$ de su área, así como también se visualiza vegetación sana en el $25 \%$ del área de un cultivo ubicado al Noroeste de la salida gráfica. En este sentido es importante aclarar, que aproximadamente seis cultivos ubicados en este mapa, presentan baja sanidad vegetal, debido a que en dichas áreas se estaban realizando en su momento, trabajos de labranza para preparación del terreno a cultivar.

En los otros cultivos de los 3 mosaicos del sector 4, es necesario desarrollar análisis de suelos para detectar la causa que genera tan baja sanidad en la vegetación con menos valores de NDVI reflejados en los mapas.

\section{CONCLUSIONES}

Las salidas gráficas generadas en este trabajo son un importante insumo que les permitirá a los agricultores asociados al Distrito de Riego, tener un diagnóstico claro sobre el estado sanitario de sus cultivos, y alertarlos sobre las áreas más críticas en las que deben prestar especial cuidado con el fin de que puedan adelantar diagnósticos más completos relacionados con estudios de suelos puntuales, a partir de los cuales puedan tomar las mejores decisiones para adaptar sus cultivos, administrar eficientemente los mantenimientos a los mismos y aumentar la productividad de estos. En este sentido, si es importante resaltar que de las 79.02 hectáreas analizadas, aproximadamente un $30 \%$ de los cultivos poseen un estado sanitario de su vegetación favorable, mientras en los demás cultivos, hay que entrar a detallar cada situación en particular, aplicando diferentes tecnologías para desarrollar agricultura de precisión que permita planificar acertadamente el desarrollo de cada cultivo.

\section{AGRADECIMIENTOS}

Los autores agradecen a Colciencias, quién financió el $60 \%$ del proyecto del cual se obtuvieron los resultados de este artículo, bajo convocatoria de jóvenes investigadores e innovadores de 2017. Además, agradecen a la Universidad Francisco de Paula Santander Ocaña por todo su patrocinio, con el aporte del $40 \%$ restante del proyecto antes mencionado.

\section{REFERENCIAS}

Burgos-Artizzu, X. P., Ribeiro, A., \& De Santos, M. (2007). Controlador borroso multivariable para el ajuste de tratamientos en agricultura de precisión. RIAI - Revista Iberoamericana de Automatica E Informatica Industrial, 4(2), 64-71. https://doi.org/10.1109/TSMC.1973.5408575

C Ferrin, X Magdalena, H Loaiza, (2017). Sistema 
de extracción automática de parámetros morfológicos de la huella plantar mediante técnicas de visión por computador en un sistema embebido. Revista Colombiana de Tecnologías de Avanzada, ISSN: 1692-7257

C Hernández, B Contreras, C Torres (2016). Desarrollo de libros electrónicos: "taller pedagógico". Revista Colombiana de Tecnologías de Avanzada, ISSN: 1692-7257.

Fischer, J. W., Greiner, K., Lutman, M. W., Webber, B. L., \& Vercauteren, K. C. (2019). Use of unmanned aircraft systems (UAS) and multispectral imagery for quantifying agricultural areas damaged by wild pigs. Crop Protection, 125, 104865. https://doi.org/10.1016/J.CROPRO.2019.104 865

Gillan, J. K., McClaran, M. P., Swetnam, T. L., \& Heilman, P. (2019). Estimating Forage Utilization with Drone-Based Photogrammetric Point Clouds. Rangeland Ecology \& Management, 72(4), 575-585. https://doi.org/10.1016/J.RAMA.2019.02.00 9

Gonzaga-Aguilar, C. (2015). Aplicación de índices de vegetación derivados de imágenes satelitales para análisis de coberturas vegetales en la provincia de Loja, Ecuador. CEDAMAZ, 5(1).

JEG Plaza, MAR Nuñez, (2017) Formación en competencias específicas para la industria del software colombiano. Experiencias del uso del aprendizaje basado en proyectos. Revista Colombiana de Tecnologías de Avanzada, ISSN: 1692-7257

Kharuf-Gutierrez, S., Hernández-Santana, L., Orozco-Morales, R., de la C. Aday-Díaz, O., \& Delgado-Mora, I. (2018). Análisis de imágenes multiespectrales adquiridas con vehículos aéreos no tripulados en agricultura de precisión. Revista Científica de Ingeniería Electrónica, Automática Y Comunicaciones ISSN: 1815-5928, 39(2), 79-91.

L Tangarife, M Sánchez, M Rojas (2017). Modelo de interventoría de tecnologías de información en el área de conocimiento de la gestión del alcance de PMBOK® y alineado con ISO 21500 y COBIT®. Revista Colombiana de Tecnologías de Avanzada, ISSN: $1692-7257$
Maes, W. H., \& Steppe, K. (2019). Perspectives for Remote Sensing with Unmanned Aerial Vehicles in Precision Agriculture. Trends in Plant Science, 24(2), 152-164. https://doi.org/10.1016/J.TPLANTS.2018.11. 007

Mogili, U. R., \& Deepak, B. B. V. L. (2018). Review on Application of Drone Systems in Precision Agriculture. Procedia Computer Science, 133, 502-509. https://doi.org/10.1016/J.PROCS.2018.07.06 3

Petzl, J., Quiroga, C., Rodríguez, A. C., Rojas, J. G., \& Ojeda, D. (2015). Paisajes del despojo cotidiano: acaparamiento de tierra y agua en Montes de María, Colombia. Revista de Estudios Sociales, (54), 107-119.

Salinas-Zavala, C. A., Martínez-Rincón, R. O., \& Morales-Zárate, M. V. (2017). Tendencia en el siglo XXI del Índice de Diferencias Normalizadas de Vegetación (NDVI) en la parte sur de la península de Baja California. Investigaciones Geográficas, (94), 0-0. https://doi.org/10.14350/rig.57214

Tello, J., Gómez-Báguena, R., \& Casterad, M. A. (2017). Comparación y ajuste en zonas agrícolas de índices de vegetación derivados de Landsat- 8 y Sentinel-2 Comparison and adjustment in agricultural areas of vegetation indexes derived from Landsat-8 and Sentinel-2. In L. A. Ruiz, J. Estornell, \& M. Erena (Eds.), Nuevas plataformas y sensores de teledetección. XVII Congreso de la Asociación Española de Teledetección (pp. 81-84). Murcia: Centro de Investigación y Tecnología Agroalimentaria de Aragón [CITA]. 